\title{
Content Management System of Website-Group Based on ZSH Frame
}

\author{
Su-qi Zhang ${ }^{1}$, Yong-feng Dong ${ }^{2}$, Bo-ying Liu ${ }^{2}$ Jun-hua Gu ${ }^{2}$ \\ ${ }^{1}$ School of Electronic Information Engineering, Tianjin University, Tianjin 300192, China \\ ${ }^{2}$ School of Computer Science and Engineering, Hebei University of Technology, Tianjin 300401, China \\ dongyf@hebut.edu.cn
}

\begin{abstract}
With development of informatization construction, it is difficult to form a unified framework among different systems, used to share information, modify and maintain. So the development of Information in a certain extent is hampered . It is presented of the more effective website group in order to adapt to the pace of informatization. The content management system (CMS) of website-group is researched and designed based of $\mathrm{ZK}+$ Spring + Hibernate $(\mathrm{ZSH})$ framework. The centralized management and distribution authorized is implemented. The information sharing and push between different websites is used to eliminate information island. It provides highly personalized show and management environment to make users self-help customization and management . the cost can effectively been reduced by sharing of software and hardware resource.

Keywords-website-group; content management system(CMS); information sharing; ZSH
\end{abstract}

\section{Introduction}

With the depth development of domestic electronic commerce and e-government, portals website played an irreplaceable role in the effective integration of the information resources. But with the deepening of the construction site, the closed building mode of existing website greatly restricted the further development of portals website and the pace of the informatization ${ }^{[1]}$. Each website can't realize the effective information sharing, formed an "information island". It leads to the low information utilization rate between each department, data redundancy, content is disagreement and many other drawbacks.

In order to further improve the portals website's construction level of government departments and enterprises, accelerate website construction, avoid repetitive construction, redundant plan, construction standard inconsistent and so on questions, we must integrate website information which is from all levels of scattered websites. It also becomes the primary requirements of portals website construction ${ }^{[2]}$. It is imperative to establish the website group management platform which can provide the unified management, the unified planning, the uniform standard, and the unified deployment to websites. This platform must be based on content management system and comprehensive use of various technologies to realize the information sharing and pushing between each website, raise the website management level and realize the low consumption to be highly effective of website construction.

\section{Technical Architecture of Website Group}

$\mathrm{ZSH}$ is the organic integration of ZK, Spring and Hibernate; it belongs to the brand-new technical framework. $\mathrm{ZK}$ is responsible for the display of system interface and the logic control. Hibernate is responsible for database operation and it is able to support many databases, this characteristics can make our systems own the enormous promotion in the performance and the expansibility. Spring provides unified management to various interfaces, this can make the system extension becomes extremely convenience. The system uses the brand-new permissions framework in the aspects of system safety. It absorbs the essence of world famous Acegi permissions framework and ensures the convenience of the system security access and the function expand.

$\mathrm{ZK}$ is an event-driven framework which is based on component and uses to enrich user interface in network procedures. ZK includes an event-driven engine which is based on AJAX, a set of XUL, XHTML and a ZUML (ZK User Interface Markup Language) markup language ${ }^{[3]}$. ZK has the rich module, so it can easily realize tree, graph, page partial dynamic variation, label page and other functions which are difficult to achieve by ordinary web procedure. It is a good tool to realize the rich client application. The mode of development for ZK is simply, we only need to master each component's application, and then it will be possible with ease to complete the application development.

Spring is developed to solve the complexity of enterprise application. From the perspective of simplicity, testability and loose coupling, any Java applications can benefit from Spring ${ }^{[4]}$. In simple terms, Spring is a vessel framework which is lightweight control reversal and faces the section.

Hibernate is an object relationship mapping framework which is open source, it makes a very lightweight object encapsulate to JDBC. Hibernate can be used in any where JDBC is used, it can be used either in Java client program or in Servlet/JSP Web applications. Figure 1 is the technical architecture of this system. 


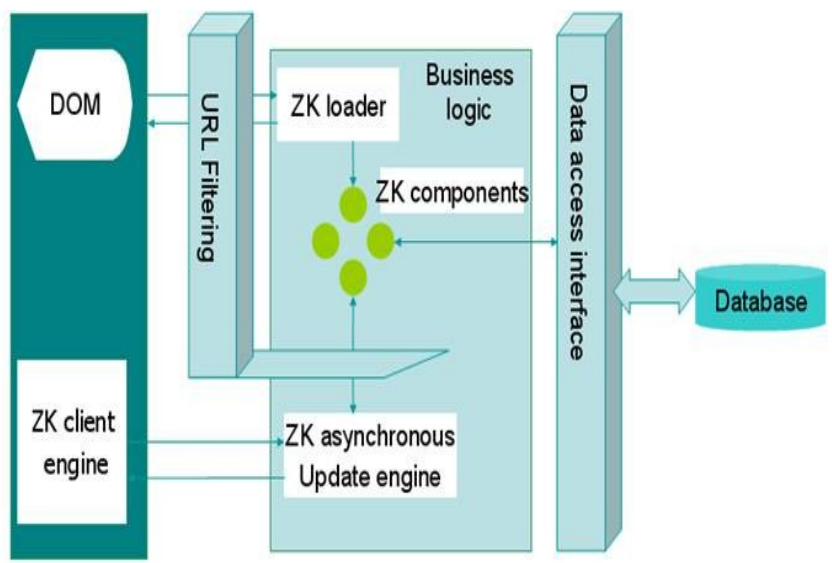

Figure 1. System technical architecture

\section{The Design of System}

A. The System Structure

The content management system of website group includes the system management, the website group management, the overall columns management, the template management, the information management, the application management and so on. The website group management provides the global management service to the configuration information of all websites, including the main portal website and other subordinate websites. The website system presents the subordinate structure, unified management top-down. But each website is relatively independent, each website has one or more domains independently and their own website management. Column is the display and navigation structure of website's information and content, and it is the classification node of website's information and content. The column can have the fathers and sons relationship, can achieve infinite levels and can form columns structure tree. We can control the column's display through the template in each column. On the one hand template defines the form and style of eventual HTML file; on the other hand it also defines the way of the data presented in the final HTML files. HTML in the templates defines the form and the style, the tag in the templates defines the data presentation. It decides to show what kind of information and how to display in some area of the template. The system supports visualization template operation and the manifestation is rich. The information can be shared to relevant column, and can also quote the information which other columns issued. The system uses the method of dynamic and static page combination to improve access efficiency and guarantees the security and stability of the system. The powerful function of statistical analysis is advantageous for the track of popular topic and plays an auxiliary function in performance assessment. Figure 2 is the whole system structure. Figure 3 is the structure of content management system of website group.

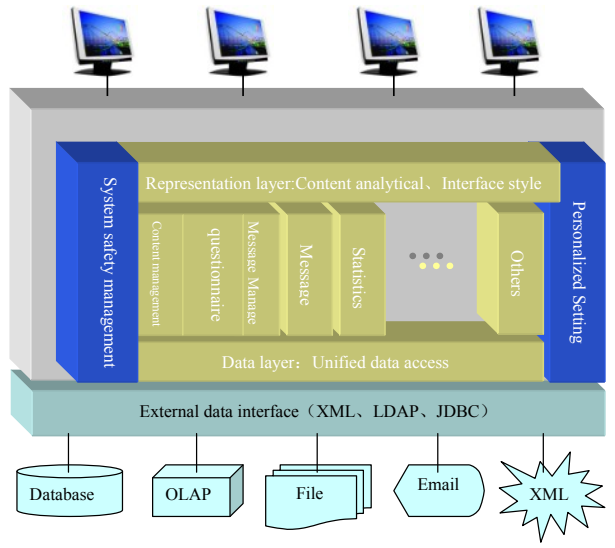

Figure 2. The whole system structure

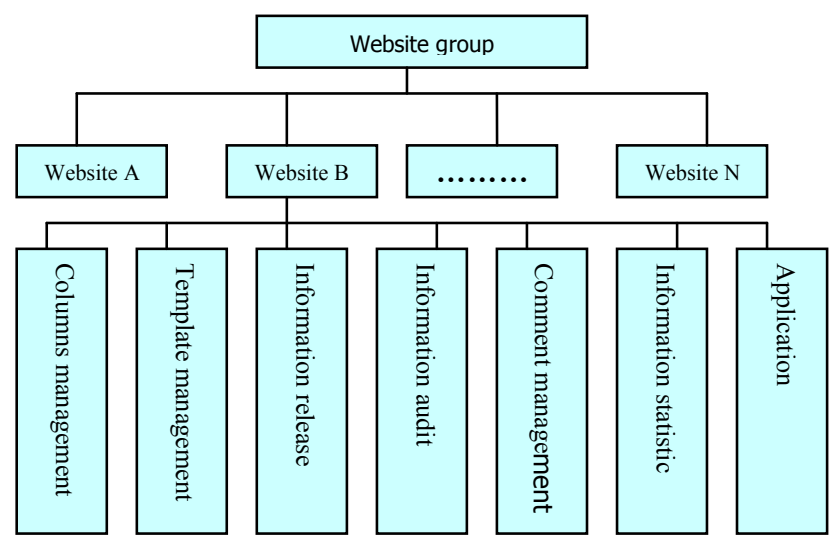

Figure 3. website group of content management system structure

\section{B. The Characteristics of System Function}

\section{1) Built website quickly and convenient operation}

The system supports drag-and-drop operation. All elements in pages like columns, pictures and so on can use the mouse to arbitrary drag. The mode of what you see is what you get gives users with extremely good operation experience and working efficiency.

The system supports the right-click menu operation. Users can make website online just like operating the Windows interface without entering tedious menu systems step by step. So the operate is very convenience.

With a complete website as a template, we can build other websites quickly through copying the complete website. We can create specified amount of websites with the function without the manual intervention.

\section{2) The website manifestation is rich}

There are many kinds of website layout template. The system contains the current popular website layout way. It's available when create the site. Rich columns style, system also contains hundreds of column's styles. According to the need of each website columns, we can designate corresponding style or customize column style online for each column. 
Changeable manifestation of information. We can set columns to display as list of headlines, scrolling marquee, data form and so on. It embodies the most representative expression form in web design.

\section{3) The highly information shares}

The system may realize the subordinate information longitudinal transfer between each website and horizontal transfer between the same level website. The information in each column of the website can realize information's forwarding through the data mapping function. The host website can send a notice to all subordinate websites; the child website can also report local information to the host website. It realizes the cross websites information's transmission and audit.

\section{4) Supporting the static page}

The system can use the method of forming static page to improve the website visit performance dramatically.

\section{5) Zero code}

The system can build large-scale website or website group without writing any codes and HTML tags. The requirement is quite low to manager's technical level.

\section{6) Supporting user custom}

Users can customize the template of page layout, columns types, columns style, and content demonstration template. The user rich imagination becomes a reality by comprehensive custom.

\section{7) Permissions management based on the roles}

The system supports the role definition. It can combine each slight power unit into any function role according to the user's need. This can make the system management more safe and convenient.

\section{8) Comprehensive statistics analysis function}

The system provides the visit analysis function of website group, it can analysis the access number of websites, columns and news. The workload statistics functions can also statistics the number of information which the manager or the correspondents issued.

\section{System Security Mechanism}

The security system includes three parts: authentication, authorization and information security defense system.

\section{A. Unified Identity Authentication and Single Point Landing}

The system uses the user identity to be only and the mode of assign roles to manage personnel permissions. Considering the conveniences and expansibility in the future, this system uses the single-point login system which can cross servers and applications. The user can be authorized all authority system only login one time through the single-point login system.

\section{B. Authorization Mechanism}

The system uses hierarchical authorized and access control strategies to strictly control the illegal user access the limited information.

\section{1) Grading authorized}

The system adopts the graduation assignment authorization mechanism. The manager of superior website can assign the permissions of lower website, such as the scope of information management, the scope of personnel management and the use scope of functional components. What's more, the manager of lower website can assign the permissions within his scope to lower site.

After the manager of superior website specify the scope of information to lower website, the lower website can develop its information and these informations naturally belong to the superior website. This mode greatly reduced the workload of all levels manager's jurisdiction assignment and information maintenance, and make the management is very convenient

\section{2) Multiple permissions control mode}

The system has very strict and flexible strategy for the information access control in a specific website. The website manager can flexible set the permissions group which the information belongs, such as allowing who browse, allowing who editor and allowing who audits. Even it can carry on the limit to the IP address.

We can create multiple different permissions group in one website, and each permissions group can bind several people. Each permissions group can bind many columns to limit information range of this permission. Each permissions group can establish the IP visits definition. In such permissions group's personnel may have certain operation permissions to the article which belongs to the binding column.

\section{Conclusion}

The content management system of website group provides a unified management platform. The application of this system not only can reduce the cost of website management, save human, material and financial resources, but also can build several websites in a short time. Different from other content management system, it based on ZSH framework and can better improve the extensibility and flexibility of system.

\section{Acknowledgment}

This paper is supported by Tianjin Research Program of Application Foundation and Advanced Technology (12JCZDJC21200).

\section{References}

[1] HongBo Chen, The research of content management systems based on the portal technology,beijing: Institute of computing technology in Chinese academy of sciences, 2006.

[2] ZhenYu Jin, The analysis of the government website group construction, Informatization construction,pp.36-37,2009 (3)

[3] Chen,H, Cheng, R, ZK framework - Ajax development of actual combat, Publishing house of electronics industry, 2009.

[4] Anil Hemrajani, JAVA agile development - Spring、Hibernate and Eclipse, People's Post and Telecommunications Press. 